\title{
Modeling the critical care pathway for cardiothoracic surgery
}

\author{
Nicolas Bahou ${ }^{1}$ (D) - Claire Fenwick ${ }^{2}$ - Gillian Anderson ${ }^{1} \cdot$ Robert van der Meer ${ }^{1}$. \\ Tony Vassalos ${ }^{2}$
}

Received: 3 July 2016 / Accepted: 1 May 2017 / Published online: 16 May 2017

(C) The Author(s) 2017. This article is an open access publication

\begin{abstract}
The west of Scotland heart and lung center based at the Golden Jubilee National Hospital houses all adult cardiothoracic surgery for the region. Increased demand for scheduled patients and fluctuations in emergency referrals resulted in increasing waiting times and patient cancellations. The main issue was limited resources, which was aggravated by the stochastic nature of the length of stay (LOS) and arrival of patients. Discrete event simulation (DES) was used to assess if an enhanced schedule was sufficient, or more radical changes, such as capacity or other resource reallocations should be considered in order to solve the problem. Patients were divided into six types depending on their condition and LOS at the different stages of the process. The simulation model portrayed each patient type's pathway with sufficient detail. Patient LOS figures were analyzed and distributions were formed from historical data, which were then used in the simulation. The model proved successful as it showed figures that were close to actual observations. Acquiring results and knowing exactly when and what caused a cancellation was another strong point of the model. The results demonstrated that the bottleneck in the system was related to the use of High Dependency Unit (HDU) beds, which were the recovery beds used by most patients. Enhancing the schedule by leveling out the daily arrival of patients to HDUs reduced patient cancellations by $20 \%$. However, coupling this technique with minor capacity reallocations resulted in more than $60 \%$ drop in cancellations.
\end{abstract}

Nicolas Bahou

nicolasbahou@gmail.com

1 Department of Management Science, University of Strathclyde, Glasgow, UK

2 Golden Jubilee National Hospital, Clydebank, UK
Keywords Simulation $\cdot$ OR in healthcare $\cdot$ Patient scheduling $\cdot$ Hospital operations

\section{Background}

Golden Jubilee National Hospital (GJNH) is Scotland's flagship hospital which specialises in heart and lung services [1]. It serves the population of the whole of the West of Scotland for all adult cardiothoracic services and is the only hospital in Scotland to provide three national services for advanced heart failure, adult congenital cardiac services and the pulmonary vascular unit. It undertakes more than 2600 heart and lung surgeries per year. Demand for these services is growing due to increases in the aging population, expected to rise by $22 \%$ by 2020 [2]. However, with reductions in public sector funding there is continual pressure for efficiency savings and the identification of improvements [3].

A previous study at the GJNH was commissioned on the formation of the specialist heart and lung centre (HLC) to investigate intensive care unit (ICU) bed capacity and balancing this with theatre time [4]. The unit at the time provided elective heart and lung surgery with very few emergencies. There was a belief at GJNH that ICU was the constraint in the system and placed focus there. Thus, Bowers tested the change in utilization between theatres and the ICU for different changes in ICU capacity. He proposed that increases in capacity for ICU will be met with increased competition for theatre space, decreasing ICU utilization and diminishing increases in throughput.

Since Bowers work in 2008 throughput has grown significantly and there is currently a $12 \%$ to $15 \%$ operation cancellation rate.

This problem meant cancelled patients required rescheduling, increasing their time to surgery. This could 
increase the probability of medical complications, decrease service quality and ultimately increase operational costs. Reducing the cancellation rate could defer the building of additional facilities at extremely high cost. GJNH was thus seeking solutions to reduce cancellations without the need for additional investment. The purpose of the current study is to explore patient scheduling and investigate opportunities for reducing the cancellation rate while considering the performance of the whole HLC system. This pilot study was directed by the hospital management. It resulted in the GJNH becoming involved in a nationally driven optimisation initiative for hospital operations.

\section{Literature review}

\subsection{Patient scheduling}

Literature on the subject of patient scheduling in hospitals is diverse in its approach to the problem [5] with the type of analysis or solution technique dependent on a number of factors. Increasing patient throughput using simple techniques is the approach taken by Litvak [6]. He makes reasonable inferences such as that increased demand cannot be resolved by individual hospitals since this is a systematic problem where an increase in the number of patients in one hospital will decrease it in another. He also mentions that increased utilization may cause increased waiting times for patients directly as a result of unpredictable (emergency) patients entering the system where an over utilized system will be forced to reschedule or cancel elective patients. Lack of credible data he suggests is one of the reasons for the underuse of simulation in hospitals. However, this is changing with the advent of electronic patient records.

In contrast, Conforti et al. [7] objective was to maximize the number of patients that undergo radiotherapy treatment by using integer linear programming. This study considered numerous constraints to arrive as close as possible to measuring the reality of the patient path including patient priority, duration of treatment and follow up outpatient scheduling. Through effective scheduling, they were able to minimize patient waiting time for treatment.

On the other hand, Arnaout and Kulbashian [8] set their objective to try and maximize the utilization of operating rooms by developing heuristic solutions and comparing them using simulation. They concluded that surgeries with longest processing time which should also include a setup time is the optimal solution for operating room utilization.

Augusto et al. [9] took a different approach in tackling hospital operation efficiency. The study challenges the rule that no patients should be allowed in the operating room if no recovery beds are available after the operation. By using a mathematical optimization model, they suggested that allowing recovery in the operating room benefits hospital operations in the circumstance that the workload of recovery beds becomes bigger than that of operating rooms.

Samudra et al. [10] reviewed many studies focused on theatre scheduling and the methodologies used. He came to the conclusion that a heuristic approach is often used whenever a problem is computationally difficult to solve within a reasonable time limit using mathematical programming. This was also the reason why a heuristic approach was used for the project at GJNH. The many different variables that were needed to make up an enhanced schedule and the stochastic nature of the HLC system were too complex to solve using mathematical programming within the time allocated for the project. In addition, a heuristic approach was seen more favourably by clinicians since it offered an easy to understand model for the purpose and scope of the project (detailed in methodology).

The discussed studies show that optimized operations and scheduling appear to be effective for a hospital, however such practices do not appear to be prevalent. Magerlein et al. [11] review the literature around surgical demand scheduling with different objectives and constraints used across different studies. Magerlein et al. mention blocked booking systems where each surgeon is scheduled a certain amount of time each week, similar to the system used by GJNH and many other hospitals nowadays [12]. This highlights that this type of scheduling is embedded and has not changed over the intervening period. Magerlein et al. later point out an article written by Stimson and Stimson (1972) who mentioned that proposed scheduling techniques fail to satisfy medical staff, where perhaps blocked booking systems are a choice that make staff more comfortable in day to day operations. This was backed by a more recent study by Guerriero [12] who mentions the importance of taking into consideration medical staff preferences while comparing Operational Research (OR) studies on surgical scheduling. Blocked booking systems was one of the obstacles that was discussed during this study at GJNH when enhancing schedules was proposed, with the hopes that a mathematical approach combined with visualizations from simulation (discussed later) will help in convincing medical staff in making some changes.

Many studies applied OR models for hospital operations but they often proved to be challenging to implement and unsustainable [13, 14]. Brailsford et al. [13] evaluated the adoption of the simulation tool similar to the one used in this study. They concluded that difficulties in adoption came from lack of capacity (lack of time, internal expertise and budget), lack of management support (due to not seeing a timely return on investment) and data issues (lack of useful data and data that hospital staff found hard to read from the software).

Other studies also suffered from problems in data collection since data could be fragmented, deficient [15] or sometimes just unavailable, forcing the modeller to make assumptions and perhaps omit important constraints that could have 
had a significant impact on the model [16]. Such studies would not be reliable since they often lack the ability to construct a meaningful model.

Other problems come from studies with contradicting results due to taking into consideration different objectives and constraints. Kwak et al. [17] and more recently Arnaout and Kulbashian [8] proposed solutions that longer operational cases should be scheduled early in the day. This is in direct opposition to the solution presented by Denton et al. [18], where they arrived at a conclusion that "the common practice of scheduling longer and more complex cases earlier in the daily schedule may have a significant negative impact on operating room performance measures". This highlights the complexity of hospital operations and the difficulty in constructing a standardized model or a common solution for the most appropriate schedule or enhanced operations in general.

Nevertheless, technologies in the field of optimization and simulation are evolving and becoming more affordable and more powerful giving them the ability to capture complex scenarios. Healthcare organizations need to engage with and understand these capabilities to realise the benefits that can be had from their use, whether exploratory or for predictive purposes.

\subsection{Simulation}

Many authors have used simulation to solve complex hospital operations problems with compelling results [19-24]. Recent advances in capturing large data is carving the way for such advanced analytical tools. For instance, in recent years, Business Intelligence (BI) tools, have become much more dominant in the business world, making them almost a necessity for every business as they offer compelling visualization and valuable insights. However, most BI tools have the limitation of only showing actual business practices and have limited capabilities for predictive, 'what if' scenarios, which only makes them useful for short to medium term decision making. Simulation models on the other hand will take the strategic analysis approach, giving insight to the future and providing long term solutions. Arguably, some of the decisions taken by only performing data analysis using BI tools could have negative consequences, since solutions will rely on people's intuition and proven techniques which might not work for the scenario at hand [25]. Simulation technology provides mathematical evidence as to what can be achieved for different scenarios, ensuring a more reliable outcome. The risk of producing a critical error is thus significantly reduced eliminating the need for rework, making it a timely and cost effective method. In hospitals in particular, performing poorly studied changes can have the detrimental effects of having to put patients' lives at risk, which is why authors are continuously clinging to simulation as the ideal method in solving hospital operations problems. Furthermore, adding a simulation model in the data analysis portfolio of hospitals (or many other organizations) provides a lifelong benefit, since simulation models can be easily altered in order to adhere to the changes made in real life. This becomes useful in dynamic operational settings proving again the effectiveness of this method in time and cost saving.

This study will use DES which uses a next-event technique where changes happen as events take place [26]. It gives the capability of following entities (patients in this case) closely in the model which is very important at logistical and operational levels [15]. Its dynamic nature and purposeful visualizations ease communication between subject matter experts and the model builder. This in turn could highlight problems in the model, providing a more accurate and visual representation of the system being modelled, essential for this study. This study used the DES software 'Simul8' (student version 1.00), which has tailored capabilities and visualizations for hospital operations.

\section{Methodology}

Determining the scope of work was one of the primary concerns of this study. Hospital operations are endlessly complex and it would be impossible to try to capture every aspect of the patients' journey and all other variables that have an effect on it. Thus, only the variables that have a direct effect on cancellation were used. The aim was to create a model that adheres to Stewart Robinsons' [27] simulation quality trilogy. This would require developing a model that conforms to the technical requirements of the study (quality of content), the expectations of the user (quality of process) and the perceived usefulness of the model (quality of outcome).

Developing the model was in direct collaboration with the scheduling department and experienced clinicians. Based on their experience and previous studies performed by GJNH it was determined that capacity was the main issue affecting the schedule and thus the model would start by patients arriving to theatres without taking the booking system into consideration.

Staff scheduling was included as a secondary objective, which determined the number of nurses needed at every change in schedule and capacity. This showed reasonable results where the demand for nurses increased as cancellations decreased. However, staffing was not fully developed in the model since it was not a main issue affecting cancellation (based on expert opinion) and thus would be omitted from further discussion.

Once the scope of work was determined, an exploratory simulation was built to capture constraints in the system which used fixed patient LOS at the different stages of the process. This was done in order to make it easier for clinicians to follow the functionality of the model before adding the complexity of variability. The next step was to add LOS distributions to the model by analysing historical data. 
The key performance indicators used to test the reliability of the model included average patient LOS in the system and the maximum number of patients in recovery beds as well as cancellations. The study then used a heuristic approach, testing the model for different schedules, capacity reallocations and different LOS for patients in order to find the scenario with minimal number of cancellations.

\section{Data analysis length of stay and theatre scheduling}

\subsection{Patient types and the critical care pathway}

In order to build the simulation model, the project started with an understanding of the inner workings of the system. Figure 1 shows the pathway of patients from the moment they enter the hospital for surgery to the moment they leave the hospital. Patients were categorised into six groups based on a variety of different factors which were agreed with the clinical team (detailed in Table 1). 'A', 'B' and ' $\mathrm{C}$ ' are cardiac patients and are defined by their LOS in the Intensive Care Units (ICU) (as shown in Fig. 4). ICUs are divided to ICU1 and ICU2 which are separately located, receiving different patient populations. 'A' patients are low risk cardiac and stay, by definition, for one day in ICU1, 'B' patients are medium risk and stay for two days in ICU1, and ' $\mathrm{C}$ ' patients are high risk and stay for three or more days in ICU2. All cardiac patients are required to stay in ICU and most of them then move to High Dependency Units (HDU), then the wards, then home. ' $\mathrm{D}$ ' and ' $\mathrm{d}$ ' are thoracic patients and are determined by the amount of time they stay in theatres. 'D' patients are major case thoracic and stay for more than two hours in theatres on average, while ' $d$ ' are minor case and stay in theatres for around one hour. Unlike cardiac patients, thoracic patients do not require ICU. 'D' patients go to HDU after surgery and 'd' patients go directly
Table 1 Description and definition of patient types

\begin{tabular}{lll}
\hline Patient type & Description & Definition \\
\hline A & Low risk cardiac & Stay for up to one day in ICU1 \\
B & Medium risk cardiac & Stay from one to two days in ICU1 \\
C & High risk cardiac & Stay for three or more days in ICU2 \\
D & Major case thoracic & Stay on average 140 min in theatres \\
d & Minor case thoracic & Stay on average 60 min in theatres \\
E & Emergency & Random arrival, stay in ICU 2 \\
& (majority cardiac) & \\
\hline
\end{tabular}

to the wards. 'E' are emergency patients and are characterised by their random arrival (this is modelled by forming a normal distribution that has a $25 \%$ standard deviation of the mean time of arrival, which accounts for high variability. Sensitivity analysis was performed by testing uniform and triangular distributions showing no significant impact on the end result), their stay in ICU2 and that they are primarily cardiac patients. Thus, similar to Bowers' [4] study, grouping was based on patient LOS and patient type, where different patients have different paths.

Figure 1 Shows demand for different patient types and number of beds at each stage. Most cardiac patients are ' $\mathrm{A}$ ' patients while most thoracic are ' $\mathrm{D}$ ' patients and there are around 45 emergency patients per year. There are four cardiac and two thoracic theatres, 10 ICU1 beds and 12 ICU2 beds. On average $21 \%$ of the available 12 ICU 2 beds are being used by patients from other departments at any one time. According to expert opinion those patients have a random arrival (for example they have the same probability of arriving 3:00 AM and 5:00 PM), and were modelled as such (similar to E patients, a normal distribution was used for their arrival with a standard deviation of $25 \%$. Sensitivity to similar input distributions was tested and had no significant effect on the
Fig. 1 Cardiothoracic patients pathway

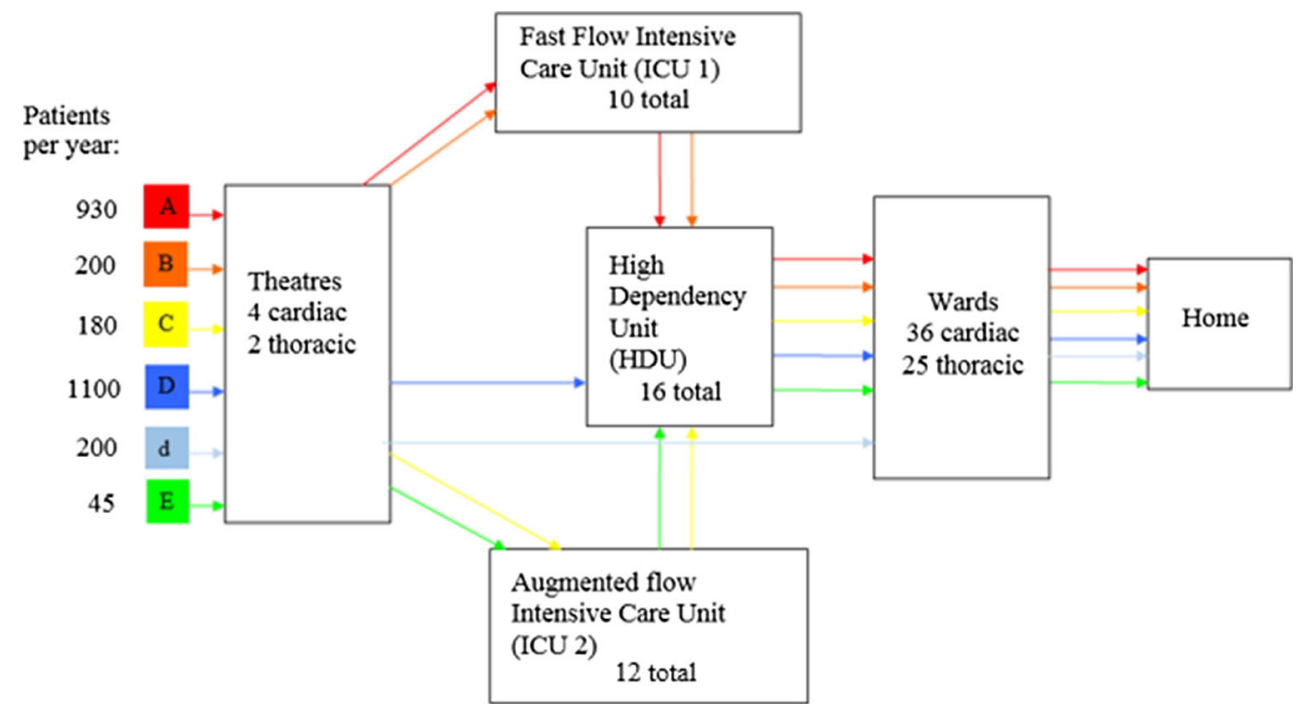




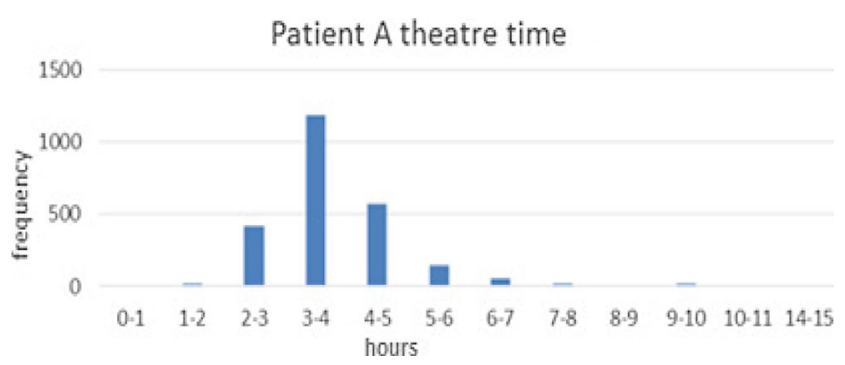

Fig. 2 'A' patients theatre length of stay

outcome nor changed the conclusions reached), where a total of 60 patients arrive to ICU2 from other departments per year. There are 16 HDU beds, 36 cardiac wards, and 25 thoracic wards.

Finally, patients were cancelled (and rescheduled) if there weren't any theatres or recovery beds for a specific patient type. For instance, 'A' patients were cancelled if all four of the cardiac theatres or if all ten of ICU1 beds were occupied. The same applied for all patients except E patients who had to be admitted within a few minutes. GJNH serves the whole population of West of Scotland and thus patients were not sent to other hospitals if they got cancelled but were slotted into the system as soon as possible pending the reason for cancellation.

\subsection{Model data}

Historical data on cardiac patients was available for all stages (theatre, ICU, HDU and ward LOS) for three years $(04 / 2012$ till 04/2015) representing the entire population of 3806 patients. On the other hand, data for thoracic patients was available for one year (from 06/2014 till 06/2015) with a representative sample of 670 patients. It also was available for all stages except theatre LOS which was based on expert opinion.

Discrete distribution representing time of day was used for patient arrival to the system (discussed in more detail in the theatre schedule section) and continuous distributions were used for every stage LOS thereafter. Figure 2 shows the distribution for ' $A$ ' patients LOS in theatres, which resembles a normal distribution. Averages and standard deviations were calculated by omitting outliers in most cases. For this distribution the averages and standard deviation of patients LOS from 1 to $7 \mathrm{~h}$ were taken, resulting in an average of around $4 \mathrm{~h}$ and a standard deviation of $1 \mathrm{~h}$. ' $\mathrm{B}$ ' and ' $\mathrm{C}$ 'patients had similar distributions with slightly higher averages for $\mathrm{B}$ patients and again higher for $\mathrm{C}$ patients.

' $E$ ' patients had a more random LOS in theatres as shown in Fig. 3. This time a triangular distribution was used. It was determined as the most appropriate pre-defined distribution since the size of data available for this specific patient type was too small to consider a more elaborate distribution. On the other hand, considering a skewed distribution here could undermine the average LOS as a whole as a result of peaks in higher LOS.

\subsection{ICU}

Noticeable from ICU LOS shown in Fig. 4 are the clusters at different time intervals.

They are created as a result of patients leaving the hospital during day time. For instance, if an 'A' patient enters ICU1 at 1:00 PM, he or she will need to stay overnight and leave the next morning (say 9:00 AM) or perhaps stay a little longer representing a $20 \mathrm{~h}$ or slightly longer LOS. Also note that ' $\mathrm{A}$ ' patients who enter ICU later in the day from afternoon surgeries and leave early the next morning will have a shorter LOS in ICU, hence the $14 \mathrm{~h}$ start in the cluster.

The same logic applies for ' $\mathrm{B}$ ' and ' $\mathrm{C}$ ' patients. In order to portray ICU LOS, normal distributions with different values were used, one for each day until the fourth day ('E' patients LOS in ICU was an average of 3.5 days). The zeros for the first day shown in Fig. 4 were not used for the first day distribution since they represent ' $\mathrm{A}$ ' patients that do not actually stay in ICU, the first of many noticed divergences from the normal daily operations presented in Fig. 1. Those who have zero LOS in ICU were also shown as having zero LOS in HDU which created a path from theatres directly to wards for 'A' patients in the simulation model (shown in Fig. 8). Several such revelations were found in the data, mostly in HDU LOS discussed in the next section.
Fig. 3 'E' Patients theatre length of stay

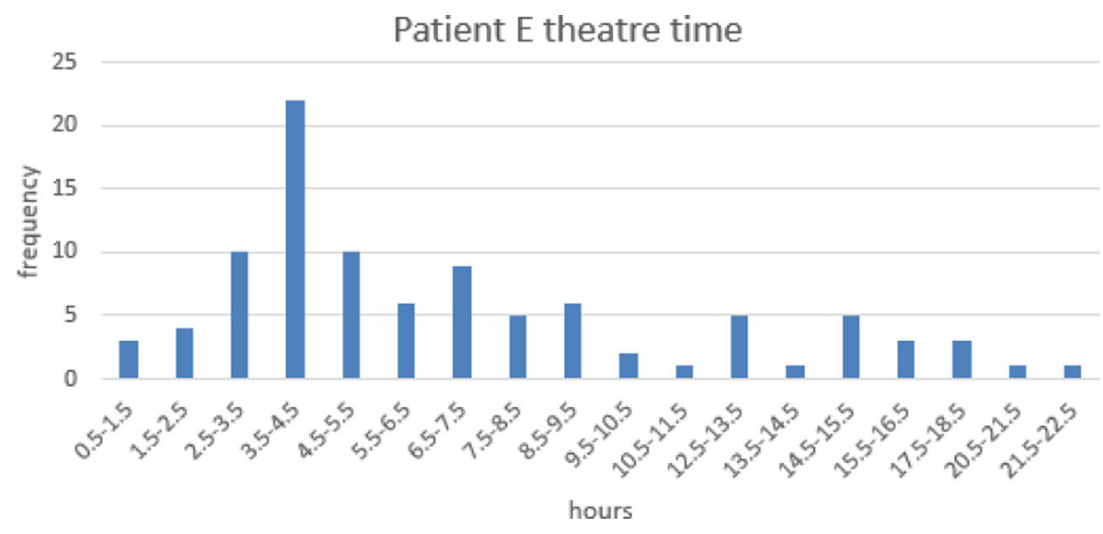




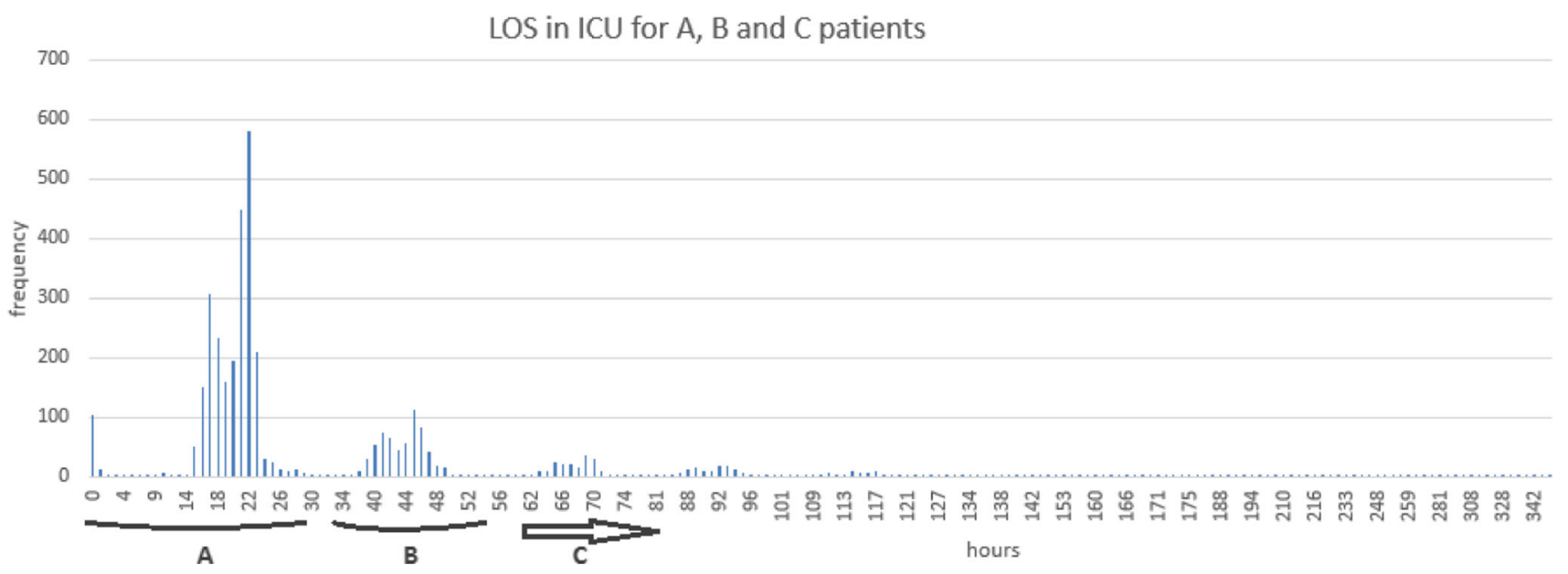

Fig. 4 ICU LOS for 'A', 'B' and 'C' patients

Clusters still existed for the fifth day onwards, but represented only $7 \%$ of the total cardiac population. Table 2 compares $\mathrm{C}$ patient LOS for three, four and five days, which shows the much lower number of patients in the fifth day compared to other days. Therefore, adding more normal distributions to represent them would make the model needlessly more complex.

Thus, as a result of the small number of data available for the fifth day onward, a triangular distribution was used. Further benefits of this distribution is that it would include all patients in the daily clusters (as opposed to a skewed distribution) and its parameters could be easily chosen to omit the outliers shown in Fig. 5. Thus, the triangular distribution acted as a uniform distribution giving a higher weight for shorter LOS as represented by the data. The minimum would thus be the start of the fifth day at $110 \mathrm{~h}$, the mode was $114 \mathrm{~h}$ and the maximum without including outliers was $1300 \mathrm{~h}$.

Table 2 Clusters of ' $C$ ' patients that stay for 3, 4 and 5 days (based on 3 years of data)

\begin{tabular}{|c|c|c|c|c|c|}
\hline \multicolumn{2}{|c|}{ Day 3} & \multicolumn{2}{|c|}{ Day 4} & \multicolumn{2}{|c|}{ Day 5} \\
\hline Hour & $\begin{array}{l}\text { Number of } \\
\text { patients }\end{array}$ & Hour & $\begin{array}{l}\text { Number of } \\
\text { patients }\end{array}$ & Hour & $\begin{array}{l}\text { Number of } \\
\text { patients }\end{array}$ \\
\hline 63 & 9 & 86 & 1 & 110 & 1 \\
\hline 64 & 9 & 87 & 6 & 111 & 7 \\
\hline 65 & 23 & 88 & 12 & 112 & 5 \\
\hline 66 & 22 & 89 & 14 & 113 & 5 \\
\hline 967 & 20 & 90 & 10 & 114 & 9 \\
\hline 68 & 16 & 91 & 9 & 115 & 7 \\
\hline 69 & 36 & 92 & 17 & 116 & 6 \\
\hline 70 & 29 & 93 & 17 & 117 & 9 \\
\hline 71 & 10 & 94 & 12 & 118 & 5 \\
\hline 72 & 5 & 95 & 3 & 119 & 3 \\
\hline 73 & 5 & 96 & 1 & 120 & 1 \\
\hline
\end{tabular}

\subsection{HDU}

Similar to ICU LOS, HDU LOS shows daily clusters (shown in Fig. 6), but this time clusters do not define patient type.

Most cardiac and thoracic patients will end up in HDU and will stay for one or more days with a certain probability as shown in Table $3(\mathrm{C} 1, \mathrm{C} 2$ and $\mathrm{C} 3$ represent $\mathrm{C}$ patients who stay for 3, 4 and 5 or more days in ICU respectively). Noticeable is that $42 \%$ of ' $\mathrm{B}$ ' patients and many ' $\mathrm{C}$ ' patients

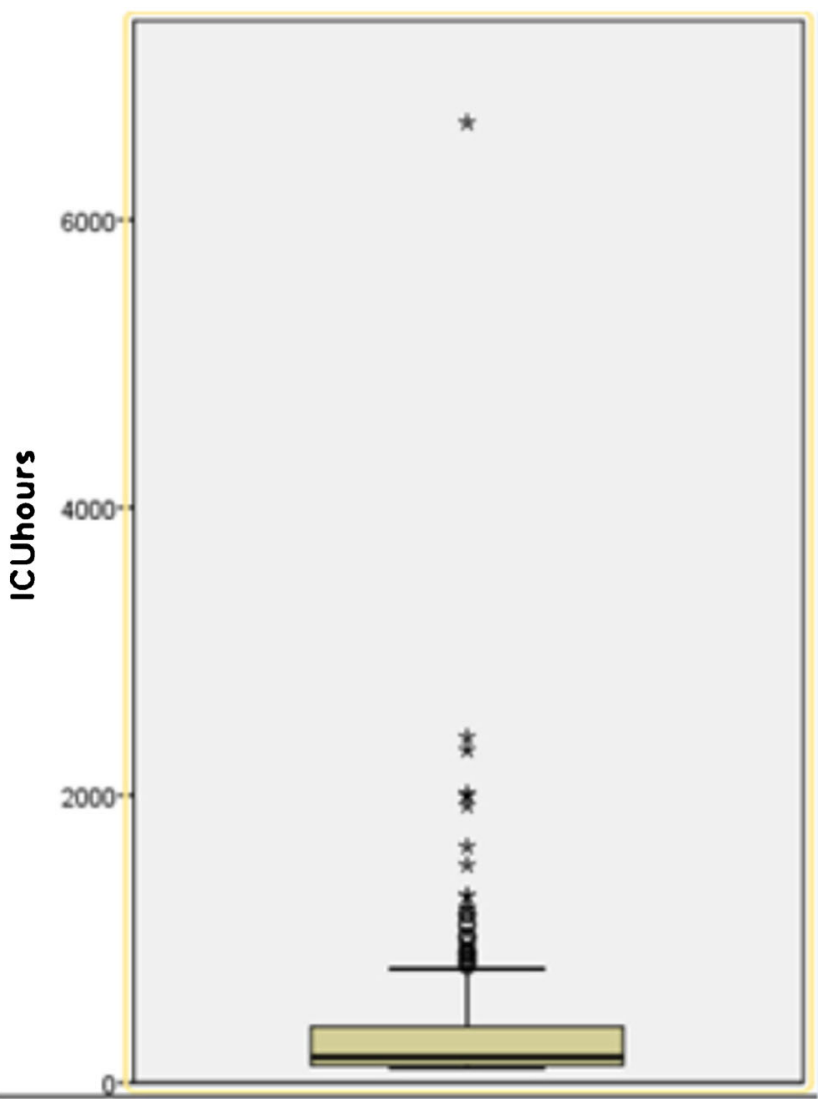

Fig. 5 ICU LOS from the fifth day onwards for 'C' patients 
Fig. 6 HDU LOS for 'A', 'B' and ' $C$ ' patients
LOS in HDU for A, B and C patients

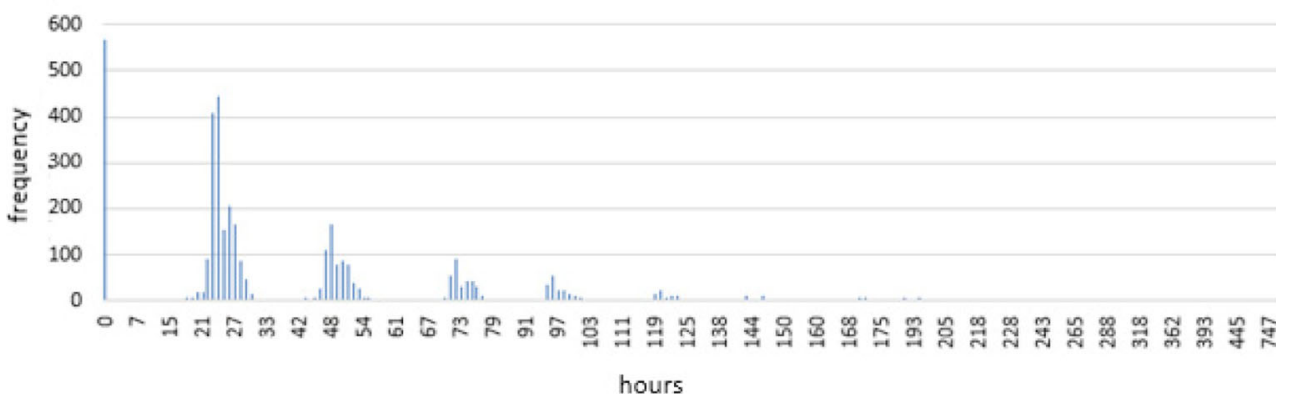

do not stay in HDU. Similar to ICU, normal distributions were used to represent daily cluster, which spans here for the first five days and for the sixth day onward a triangular distribution was used (for the same purpose as the one used for ICU) with a minimum of $137 \mathrm{~h}$, a mode of $146 \mathrm{~h}$ and a maximum of $500 \mathrm{~h}$ after omitting outliers.

\subsection{Ward}

All patients follow the same distribution for Ward LOS, where the aggregate is shown in Fig. 7. A log normal distribution was used here with an average of 6.25 days and a standard deviation of 7.7 days.

\subsection{Theatre schedule}

In order to authenticate the model with performance measures that mimic as much as possible the real numbers that GJNH record in the system (including cancellation percentages), their current schedule needed to be closely matched. The little data that was available enabled the creation of a somewhat representative schedule in the model. Theatres were open Monday through Friday 8:00 AM till 6:00 PM, with one in two Fridays closing at 12:00 noon. It showed that around two thirds of patients get scheduled before noon at 8:00 and 9:00 AM, and the rest at around 2:00 PM. 'A' patients are mostly scheduled on Tuesdays and ' $\mathrm{C}$ ' patients on Mondays.

Table 3 Percentage of patients 'A', 'B' and ' $C$ ' day stay in HDU

\begin{tabular}{lllllll}
\hline Days/patient & $\mathrm{A}$ & $\mathrm{B}$ & $\mathrm{C} 1$ & $\mathrm{C} 2$ & $\mathrm{C} 3$ & $\mathrm{D}$ \\
\hline 0 & $5 \%$ & $42 \%$ & $33 \%$ & $21 \%$ & $33 \%$ & $0 \%$ \\
1 & $56 \%$ & $23 \%$ & $20 \%$ & $29 \%$ & $17 \%$ & $56 \%$ \\
2 & $18 \%$ & $14 \%$ & $20 \%$ & $24 \%$ & $14 \%$ & $24 \%$ \\
3 & $9 \%$ & $8 \%$ & $13 \%$ & $10 \%$ & $7 \%$ & $8 \%$ \\
4 & $5 \%$ & $5 \%$ & $5 \%$ & $4 \%$ & $7 \%$ & $4 \%$ \\
5 & $3 \%$ & $2 \%$ & $2 \%$ & $4 \%$ & $3 \%$ & $3 \%$ \\
$6+$ & $4 \%$ & $5 \%$ & $7 \%$ & $10 \%$ & $18 \%$ & $5 \%$ \\
\hline
\end{tabular}

\section{The simulation model}

Figure 8 shows the simulation model, which reflects the patient pathway previously shown in Fig. 1 . The number of beds shown is not the actual number of beds but a representation of the different distributions (where one bed image can contain several patients). The arrows represent the different patient routes. For example, if an ' $A$ ' patient is admitted to surgery he or she will go to theatre ' $A$ ' (which has a LOS of the distribution shown in Fig. 3 and could accommodate up to four ' $A$ ' patients, as long as the sum of all cardiac theatres is equal to the capacity of four) and later as data suggested, have a $95 \%$ probability of moving to ICU1 beds and a $5 \%$ chance of moving to the wards. It could be noticed here the difference in complexity between the initial representation of the model (Fig. 1) and the representation from simulation and that is as a result of data analysis. It shows that there is greater variability in patient movement than might otherwise be assumed.

The simulation model runs for $24 \mathrm{~h}$ seven days a week for a whole year in order to take into consideration patient stay in the system as a whole and not theatre operating times. It also considers a warm up period, where results are only recorded after the third week. Three weeks were chosen since the biggest majority of patients will have a LOS in the whole system less than this period.

Perhaps the strongest point of the model is the way it gathers results. The most important measure captured is the exact time that a patient is cancelled. This is possible because the software is linked to an Excel file that records a cancellation as soon as it occurs, showing the type of patient cancelled and time of cancellation (for example: an ' $A$ ' patient getting cancelled at day 67, 2:15 PM). This facilitates scenario analysis with changes to the schedule in order to reduce cancellation.

All the results in the project were based on trials of 100 independent simulation runs (thus, one trial is equal to 100 runs) accounting for the warm-up period followed by a simulated year looking at the hospital activities 24/7. This is a common practice in simulation which allows to even out extreme values from single runs. The trials were based on providing a $95 \%$ confidence interval. 
Fig. 7 Ward LOS for 'A', 'B' and 'C' patients
A, B and C patient Ward LOS

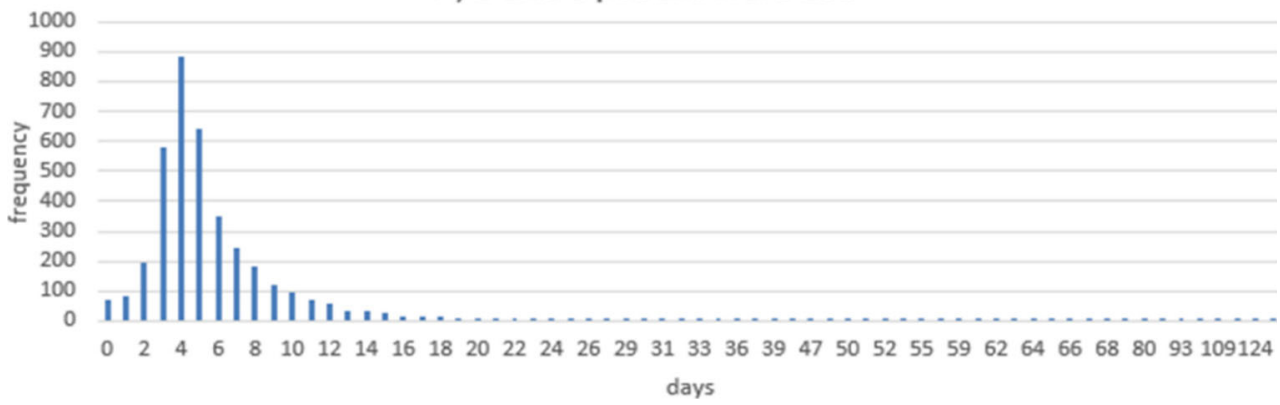

\section{Authenticating the model}

All technicalities of the model were tested on separate simpler models to check for discrepancies. Also, different LOS were tested to check for logical changes to results such as the effect of changing the maximum LOS at an ICU. The real test however came from simulating the current hospital practices using current schedule and checking the numbers from the model against actual values. This was done in conjunction with the team at GJNH. All values including average LOS and number of patient entry at different times and stages, closely resemble actual values. Most importantly however is the cancellation rates which amounted to $12.4 \%$ from a single run and between $11.7 \%$ and $15.8 \%$ with an average of $13.6 \%$ from 100 runs (of the same year) as shown in Fig. 9 (those percentages were calculated using a demand of 2670 patients, which was the demand used in the model). This represents around $95 \%$ accuracy when comparing to historical data.

\section{Running simulations and analysing results}

Extracting results for the current schedule from the Excel file revealed that most cancelled patients were ' $\mathrm{D}$ ' patients and that they were mostly cancelled on Wednesdays and Thursdays as shown in Table 4. Also, the results in the simulation revealed that more than 300 ' $\mathrm{D}$ ' patients were cancelled due to lack of HDU space, making it the clear bottleneck in the system.

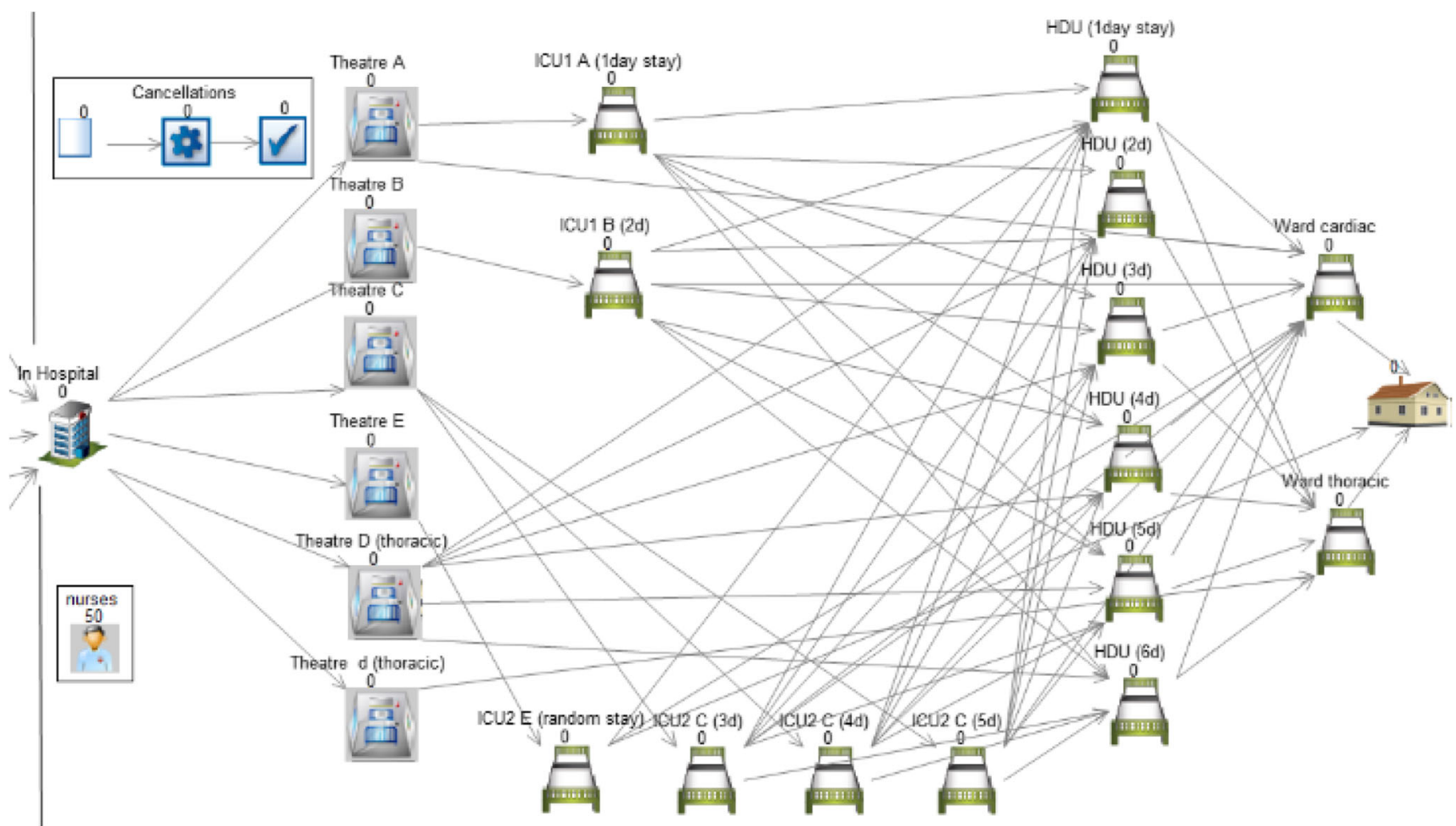

Fig. 8 The simulation model 


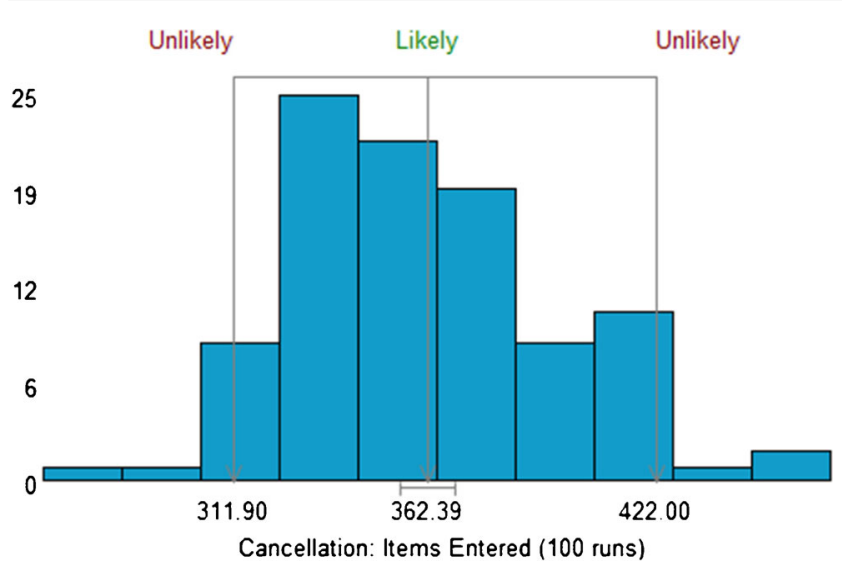

Fig. 9 Results from 100 runs of current schedule

To further back this, a sensitivity analysis was performed in order to know the effect of reduced ward space to ensure that wards would not eventually block patients in HDUs (shown in Fig. 10). In the model, patients were not getting blocked from entering wards, however the reason could be that, since the data provided is the actual LOS of patients, the LOS could reflect patients staying longer due to blocking in real life. This would suggest that a small change in ward space should have a direct effect on cancellation. Since cardiac and thoracic patients can use beds on each other's wards (only when there is no other option), the total number of beds (61) was taken into consideration. Figure 10 clearly shows that the removal of the first two beds does not have any effect on cancellation and it is only after removing the 9th bed that cancellations start to increase significantly. Thus, it could be safe to assume that HDU is indeed the bottleneck in the system.

Going back to Bowers' study, the limitation of only considering the ICU and theatres is clear. The results here might explain why increasing ICU capacity considerably in Bowers' study only resulted in a slight increase in HLC throughput. Nevertheless, some further analysis is needed to see the correlation between results from both papers.

Table 4 Patients cancelled on specific days

\begin{tabular}{lllllll}
\hline Day/Patient type & A & B & C & D & d & Grand Total \\
\hline Monday & & & & 8 & 8 \\
Tuesday & & 3 & & 55 & 1 & 59 \\
Wednesday & & & & 123 & 1 & 124 \\
Thursday & 1 & & 4 & 96 & & 101 \\
Friday & & & & 46 & 46 \\
Grand Total & 1 & 3 & 4 & 328 & 2 & 338 \\
\hline
\end{tabular}

\subsection{Enhancing the schedule}

The method used in order to enhance the schedule was to run simulations and analyse results from excel files to understand when and what causes patient cancellation and adjust numbers in schedules accordingly. After several trial and errors the schedule that produced the least cancellation with the time span of the project reduced cancellations by $20.7 \%$. This was achieved by diverting cardiac patients from reaching the HDU at peak times, especially in the middle of the week and thus allowing their recovery on weekends. Hence, 'A' patients were scheduled mostly on Thursdays and Fridays, since it takes them one day in ICU before reaching HDU. The method for other patients was the same. 'B' patients were scheduled on Wednesdays and Thursdays (since all Friday spots were taken by 'A' patients) and ' $\mathrm{C}$ ' patients were mostly scheduled on Tuesdays and Wednesdays. A small number of 'A' patients were scheduled on Mondays. As for ' $\mathrm{D}$ ' patients, since they are many and can only be scheduled in batches of two (to adhere to the theatre constraint), they were scheduled nearly equally for all days of the week, but slightly less by the end of the week, on Wednesdays, Thursdays and even less so on Fridays. This caused cancellation to be more evenly distributed throughout the week with Wednesday still having the highest numbers at however a much more acceptable 67 patients total.

\subsection{Other techniques}

It would be interesting at this point to know the effect of increasing the capacity of HDU. In order to save cost, this will entail removing the beds from another resource. Several trials of the model showed that the most appropriate capacity reallocation solution was to move two ICU1 beds to HDU. This resulted in a decrease of $46.4 \%$ cancellation under current schedule and $57.5 \%$ cancellation under the enhanced schedule (As shown in Table 5 which shows all the results for all the trials performed on the model). The $57.5 \%$ was achieved without enhancing it for this specific layout and thus a lower cancellation could be further achieved. ' $D$ ' patients remained the patients that get cancelled the most by far, however cancellation for ' $A$ ' and ' $B$ ' patients increased slightly.

Other techniques were used to lower cancellation even further and the best technique achieved with the time given for the project, was to treat all ' $\mathrm{A}$ ' patients as ' $\mathrm{B}$ ' patients with a specific schedule enhanced for this method. This was a reasonable consideration since ' $\mathrm{B}$ ' patients stay on average for a slightly longer period of time in recovery beds and they stay for longer in ICU. Thus, treating 'A' patients like 'B' patients will allow for better care for ' $\mathrm{A}$ ' patients. As a result, this solution could be implemented as part of an enhanced recovery program, but at an extra cost, since managing ICU is more expensive than managing HDU. Nevertheless, this method 
Fig. 10 Ward sensitivity

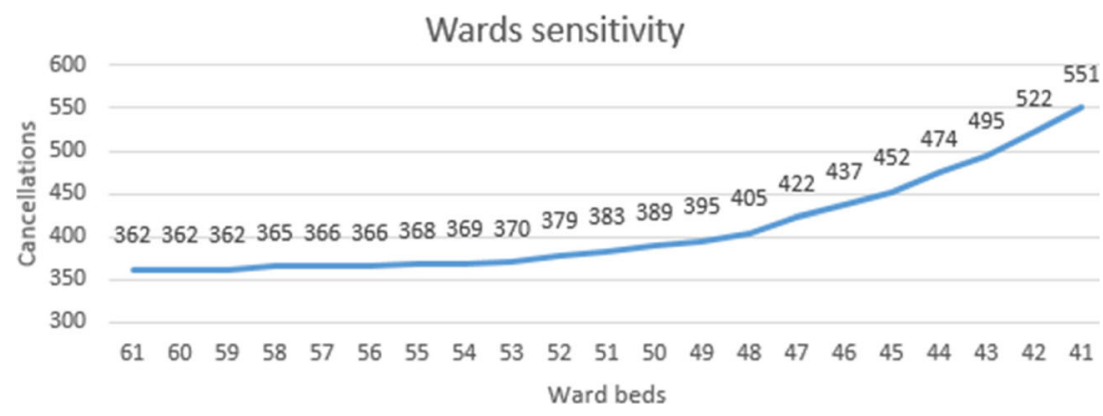

reduced cancellation by more than $60 \%$, making it an option worthy of management consideration, especially if the costs of managing the ICUs could be offset by the costs of reduced cancellations. Using this method caused more ' $A$ ' patients to be cancelled reaching 30 by the end of the year, and ' $D$ ' patients remained the patients that get cancelled the most with 58 cancellations.

\section{Discussion}

\subsection{Model advantages}

The study explored the effects of enhanced scheduling on patient cancellation using DES. The final model provided all the benefits of simulation including a risk free, time effective and a flexible way to enhance operations while reflecting a considerably accurate representation of actual operations. The objective was to reduce cancellation which has the effect of increasing throughput, agreeing to the claims made by Litvak [6]. Increasing demand is a systematic problem while increasing utilization could mean additional blockages in the system and additional cancellations. Increasing throughput is the only solution that solves the problem of the risk of complications to patients' condition and also reduces the administrative costs of having to reschedule.
The study used Simul8, which has the benefit of following individual entities with a visual element which makes communication of the model to stakeholders easier. This has proved very useful as clinical staff who were unfamiliar with DES modelling were able to follow and understand the logic of the model which allowed them to give useful input as the project progressed. The numbers in the simulation model closely resembled those seen in real life which helped increase the confidence in its outcomes. The model was fairly easy to construct since its purpose was well defined, most of the data needed for its construction was available and reliable, and most importantly there was high involvement between clinicians and the model builder. This highlights the fact that any hospital that has access to similar data should be able to replicate the model. Following the success of the project, the hospital joined a national project run by the Institute for Healthcare Optimization (ScotPFA: guided patient flow analyses) which will address every component of the patient journey.

\subsection{Model limitations}

Despite its obvious advantages, the model still had some limitations which are common to simulation in general. This includes the amount and type of information that the software can process, and the judgements involved in deciding what information to include. This is aggravated by the complex
Table 5 Cancellation percentages of different scenarios

\begin{tabular}{llll}
\hline Scenario & Cancellation & $\begin{array}{l}\text { Cancellation } \\
\%\end{array}$ & $\begin{array}{l}\text { Cancellation } \\
\text { reduction } \%\end{array}$ \\
\hline Current schedule and capacity & 362 & $13.6 \%$ & - \\
Current schedule, moving one ICU1 bed to HDU & 271 & $10.1 \%$ & $25.1 \%$ \\
Current schedule, moving two ICU1 beds to HDU & 194 & $7.3 \%$ & $46.4 \%$ \\
Current schedule, treating all A as B patients & 150 & $5.6 \%$ & $58.6 \%$ \\
Enhanced schedule, current capacity & 287 & $10.7 \%$ & $20.7 \%$ \\
Enhanced schedule, moving one ICU1 bed to HDU & 201 & $7.5 \%$ & $44.5 \%$ \\
Enhanced schedule, moving two ICU1 beds to HDU & 154 & $5.8 \%$ & $57.5 \%$ \\
Enhanced schedule, treating all A as B patients & 180 & $6.7 \%$ & $50.2 \%$ \\
Enhanced schedule specific for treating all A patients & 142 & $5.3 \%$ & $60.7 \%$ \\
$\quad$ as B patients & & & \\
\hline
\end{tabular}


structure of hospital operations [28]. For instance, dividing patients into different types comes as a result of the difficulty in taking into consideration all patient illnesses both in terms of software capability and the ability to form a valid or accurate model. This proved to be a limitation since the model showed that a change in patient type after admission (which is incorporated in the model but is not shown in Fig. 8) had a direct effect on cancellation. Thus, in terms of scheduling, the model is only as good as determining the characteristics that form the different patient types before being admitted. This will require a different study from GJNH which would classify patients depending on the expected time they would stay in recovery beds with a minimal chance of error. This could then be easily reflected in the model (where the chance of error is taken into consideration), giving a more accurate result of the effects of enhanced scheduling.

Therefore, enhancing schedules might still seem a difficult task even with this study. Nevertheless, the solutions of capacity reallocations and treating all ' $\mathrm{A}$ ' patients as ' $\mathrm{B}$ ' patients (just keep all who enter ICU1 for two days) will not be effected by the lack of patient type information. However, coupling those solutions with improved scheduling proved effective and thus a subject for further study.

Other limitations could have come from the high level of variability in the model especially from the random arrival of emergency patients and random arrival of patients from other departments to ICU2, which resulted in high fluctuations in cancellations from different runs. This is essentially why all results shown in this study are based on the averages of 100 runs, which smooths out extreme values of cancellations (low or high) from single runs.

Also, the assumption of the different continuous distributions might not be very accurate. This was minimized by testing the model for more extreme values for the same LOS. For instance, different maximum times for ICU and HDU stays were tested and proven to not have a detrimental effect on the simulation model.

There could be other questions related to limitations that the reader may have. For instance, why aren't cancelled patients re-entering the simulation? The problem in doing this is that adding such a layer of complexity will require additional data on the time before cancelled patients get back to surgery. It also adds complication in building the model to enable reentry of patients at specific times that would otherwise not be occupied by patients already scheduled. A simple way around this was assuming the demand of patients as opposed to the actual number that enter surgery by the end of the year. Another question may relate to patients blockage in the system which causes a longer stay in the model than in reality. However, the simulation model gives priority for patients to be admitted if they were to get blocked, resulting in almost $0 \%$ blocking throughout the model and an average time in the model that is close to what is observed in real life.

\section{Conclusion}

Increasing supply demand mismatch of patients admitted to the west of Scotland heart and lung centre prompted managers to review this highly valued national service. Feeding actual hospital LOS data to a simulation model proved effective in measuring the results of applying different scenarios to hospital operations including enhancing the schedule, reallocating capacity and also enhancing the recovery of different patients at the different stages of their stay (treating all 'A' patients as ' $B$ ' patients in this case). Building an enhanced schedule resulted in an acceptable $20.7 \%$ reduction in cancellation, however combining this method and changing the LOS of a certain patient types at different stages caused cancellation to be reduced by more than $60 \%$.

Discrete event simulation and Simul8 in particular produced visualizations that helped the parties involved in the project to communicate and understand both the model and the findings. The positive aspect came from producing results that were close to what was observed in real life and testing different scenarios in an easy and time effective way. Limitations were mainly due to limitations in capturing yet more details of the HLC process. This would require additional testing comparing the simulation to historical data. However, clearly there is still scope for further research.

The project served as a learning exercise for GJNH to understand the impact of adopting DES and would later serve as a basis for further projects and studies. Key to the successful completion of this project was the availability of data, the capabilities of the software product used and also the effective communication between all parties involved.

Open Access This article is distributed under the terms of the Creative Commons Attribution 4.0 International License (http:// creativecommons.org/licenses/by/4.0/), which permits unrestricted use, distribution, and reproduction in any medium, provided you give appropriate credit to the original author(s) and the source, provide a link to the Creative Commons license, and indicate if changes were made.

\section{References}

1. NHS Scotland (2015) National waiting times centre board: annual report and accounts

2. National Records of Scotland (2012) Projected Population of Scotland (2012 based). https://www.nrscotland.gov.uk/statisticsand-data. Accessed 14 Jan 2016

3. Audit Scotland (2012) Cardiology services, audit Scotland. http:// www.audit-scotland.gov.uk. Accessed 14 Jan 2016

4. Bowers J (2013) Balancing operating theatre and bed capacity in a cardiothoracic centre. Health Care Manag Sci 16(3):236-244

5. Cardoen B, Demeulemeester E, Beliën J (2010) Operating room planning and scheduling: a literature review. Eur J Oper Res 201(3):921-993 
6. Litvak E (2005) Optimizing patient flow by managing its variability. Front office to front line: essential issues for health care leaders. Joint Commission Resources, Oakbrook Terrace, pp 91-111

7. Conforti D, Guerriero F, Guido R (2008) Optimization models for radiotherapy patient scheduling. 4OR, 6(3), 263- .

8. Arnaout JPM, Kulbashian S (2008) Maximizing the utilization of operating rooms with stochastic times using simulation. In proceedings of the 40th conference on winter simulation (pp. 1617-1623). Winter simulation conference

9. Augusto V, Xie X, Perdomo V (2010) Operating theatre scheduling with patient recovery in both operating rooms and recovery beds. Comput Ind Eng 58(2):231-238

10. Samudra M, Van Riet C, Demeulemeester E, Cardoen B, Vansteenkiste N, Rademakers FE (2016) Scheduling operating rooms: achievements, challenges and pitfalls. J Sched 19(5):493-525

11. Magerlein JM, Martin JB (1978) Surgical demand scheduling: a review. Health Serv Res 13(4):418

12. Guerriero F, Guido R (2011) Operational research in the management of the operating theatre: a survey. Health Care Manag Sci 14(1):89-114

13. Brailsford SC, Bolt TB, Bucci G et al (2013) Overcoming the barriers: a qualitative study of simulation adoption in the NHS. J Oper Res Soc 64(2):157-168

14. Demeulemeester E, et.al (2013) Operating room planning and scheduling, in handbook of healthcare operations management, Denton BT (Ed.), p121-152, Springer

15. Marshall DA, Burgos-Liz L, IJzerman MJ, Osgood ND, Padula WV, Higashi MK et al (2015) Applying dynamic simulation modelling methods in health care delivery research - the simulate checklist: report of the ispor simulation modeling emerging good practices task force. Value Health 18(1):5-16

16. Ronen B, Pliskin JS, Pass S (2012) Focused operations management for health services organizations. John Wiley \& Sons
17. Kwak NK, Kuzdrall PJ, Schmitz HH (1976) The GPSS simulation of scheduling policies for surgical patients. Manag Sci 22(9): 982-989

18. Denton B, Viapiano J, Vogl A (2007) Optimization of surgery sequencing and scheduling decisions under uncertainty. Health Care Manag Sci 10(1):13-24

19. Brailsford SC, Harper PR, Patel B, Pitt M (2009) An analysis of the academic literature on simulation and modelling in health care. $\mathrm{J}$ Simul 3(3):130-140

20. Gandhari RKR (2015) Obstetric unit capacity optimization and consolidation analysis using simulation modeling and using analytical hierarchy process (Doctoral dissertation, The University of Akron)

21. Harper PR, Shahani AK (2002) Modelling for the planning and management of bed capacities in hospitals. J Oper Res Soc 53(1): $11-18$

22. Van Berkel PT, Blake JT (2007) A comprehensive simulation for wait time reduction and capacity planning applied in general surgery. Health Care Manag Sci 10(4):373-385

23. Mallor F, Azcárate C (2011) Combining optimization with simulation to obtain credible models for intensive care units. Ann Oper Res. doi:10.1007/s10479-011-1035-8

24. Shahani AK, Ridley SA, Nielsen MS (2008) Modelling patient flows as an aid to decision making for critical care capacities and organisation. Anaesthesia 63(10):1074-1080

25. Sterman JD (2000) Business dynamics: systems thinking and modelling for a complex world, vol 19. Irwin/ McGraw-Hill, Boston

26. Pidd M (2004) Computer simulation in management Science, 5 th edn. John Wiley \& Sons

27. Robinson S (2002) General concepts of quality for discrete-event simulation. Eur J Oper Res 138(1):103-117

28. Tako AA, Robinson S (2014) Is simulation in health different. J Oper Res Soc 66(4):602-614 